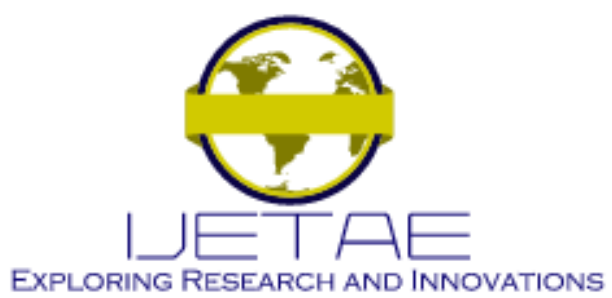

International Journal of Emerging Technology and Advanced Engineering

Website: www.ijetae.com (E-ISSN 2250-2459, Scopus Indexed, ISO 9001:2008 Certified Journal, Volume 11, Issue 11, November 2021)

Manuscript Received: 04 October 2021, Received in Revised form: 01 November 2021, Accepted: 09 November 2021

DOI: $10.46338 /$ ijetae1121_24

\title{
Linear Feedback Shift Register and its Applications in Digital System Design
}

\author{
Hai T. Nguyen ${ }^{1}$, Giao N. Pham², Anh N. Bui ${ }^{3}$, Binh A. Nguyen ${ }^{4}$, Ngoc T. Le ${ }^{5}$, Hanh T. Pham ${ }^{6}$ \\ ${ }^{1}$ Hutech Institute of Engineering, Ho Chi Minh City University of Technology (HUTECH), Vietnam \\ ${ }^{2,3}$ Dept. of Computing Fundamentals, FPT University, Hanoi, Vietnam \\ ${ }^{4,5,6}$ ICT Department, FPT University, Hanoi, Vietnam
}

\begin{abstract}
In digital system design, the Linear Feedback Shift Register (LFSR) is the queen of logic functions, and the design engineers can use LFSR in both hardware (HW) or software (SW) implementation. In this paper, LFSR will be discussed in its HW implementation via Hardware description language. In addition, the application of LFSR in of pseudorandom number generator (PRNG), direct sequence spread spectrum (DSSS), cyclic redundancy check (CRC) is also given.
\end{abstract}

Keywords-- Digital system design, System on chip, ASIC digital design, Linear feedback shift register.

\section{INTRODUCTION}

Digitization has spread to a wide range of applications around us, including from the large systems such as the information system (computers), the telecommunication system (servers), satellite system, self-driving car system, smart city system, smart house system, robotic and automation to a small system such as the mobile phone system, wearable devices [1]. To develop and design a digital system, there are some common but most important modules such as: processor or microprocessor single core or multi-cores, interrupts controller, cache, random access memory, read only memory, analogue front end controller, I/O controller, interconnect bus controller, Peripheral bus controller, analogue-to-digital, digital-to-analogue, digital signal processors [2]. Due to the level, the functional of digital system, design engineers can consider the suitable modules, memory, and bus, and some specific modules.

It has a theory that every engineer has his or her favorite fundamental designs, components, or functions. And in the digital hardware design engineering world, the Linear Feedback Shift Register (LFSR) is popular chosen in almost modules [3, 6]. In this paper, we will cover the explanation about above statements. We will organize this paper as follows, in section II, the fundamental of LFSR is given; in section III, various applications of LFSR are discussed.

\section{LFSR FUNDAMENTALS}

An n-bits Linear Feedback Shift Register (LFSR) consists of $n$ memory elements (or flops) and XOR gates.

\begin{tabular}{|l|}
\hline \\
\hline
\end{tabular}

FigURE I. BASIC ARCHITECTURE OF A LFSK SYSTEM

FIGURE I show a basic architecture of LFSR. In overall, it includes two parts: shift register and feedback network. The feedback networks relate to the mathematical models by the feedback polynomial as given in table of FIGURE I. 


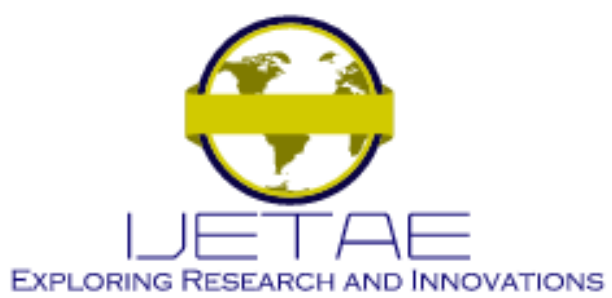

International Journal of Emerging Technology and Advanced Engineering

Website: www.ijetae.com (E-ISSN 2250-2459, Scopus Indexed, ISO 9001:2008 Certified Journal, Volume 11, Issue 11, November 2021)

\section{LFSR APPLICATIONS IN DIGITAL SYSTEM DESIGN}

\section{A. Deterministic Random Bit Generator}

DRBG or pseudo random number generator (PRNG) is the mathematical algorithm to simulate the sequence of random number or approximating the properties of random numbers. PRN generator could be used in many types of application such as Monte Carlo methods, quantum measurement, board game processors, random sampling... FIGURE II includes the overall architecture of a PRNG based on LFSR, an example with generator matrix $x^{\wedge} 4+x^{\wedge} 3+1$, hardware description language implementation, and test bench simulation.

\section{B. Direct Sequence Spread Spectrum}

DSSS is a digital modulation algorithm which is used in CDMA telecommunication system.
The advantages of DSSS are the sharing of single channel to multiple users, the reduction of background noise level, and synchronization between transmitter and receivers (via matched filter in receiver). FIGURE III includes the overall architecture of DSSS of a novel code 0x58F9A42B based on LFSR, its hardware description language implementation, and its test bench simulation.

\section{Cyclic Redundancy Check - CRC}

CRC is a detecting method which based on the mathematical model named polynomial for its generator. We can see many possible applications of CRC in many standards of communication protocols such as USB, GSM, Bluetooth, and CDMA...FIGURE IV includes the overall architecture of CRC in USB based on LFSR, its hardware description language implementation, and its test bench simulation $[4,5]$.

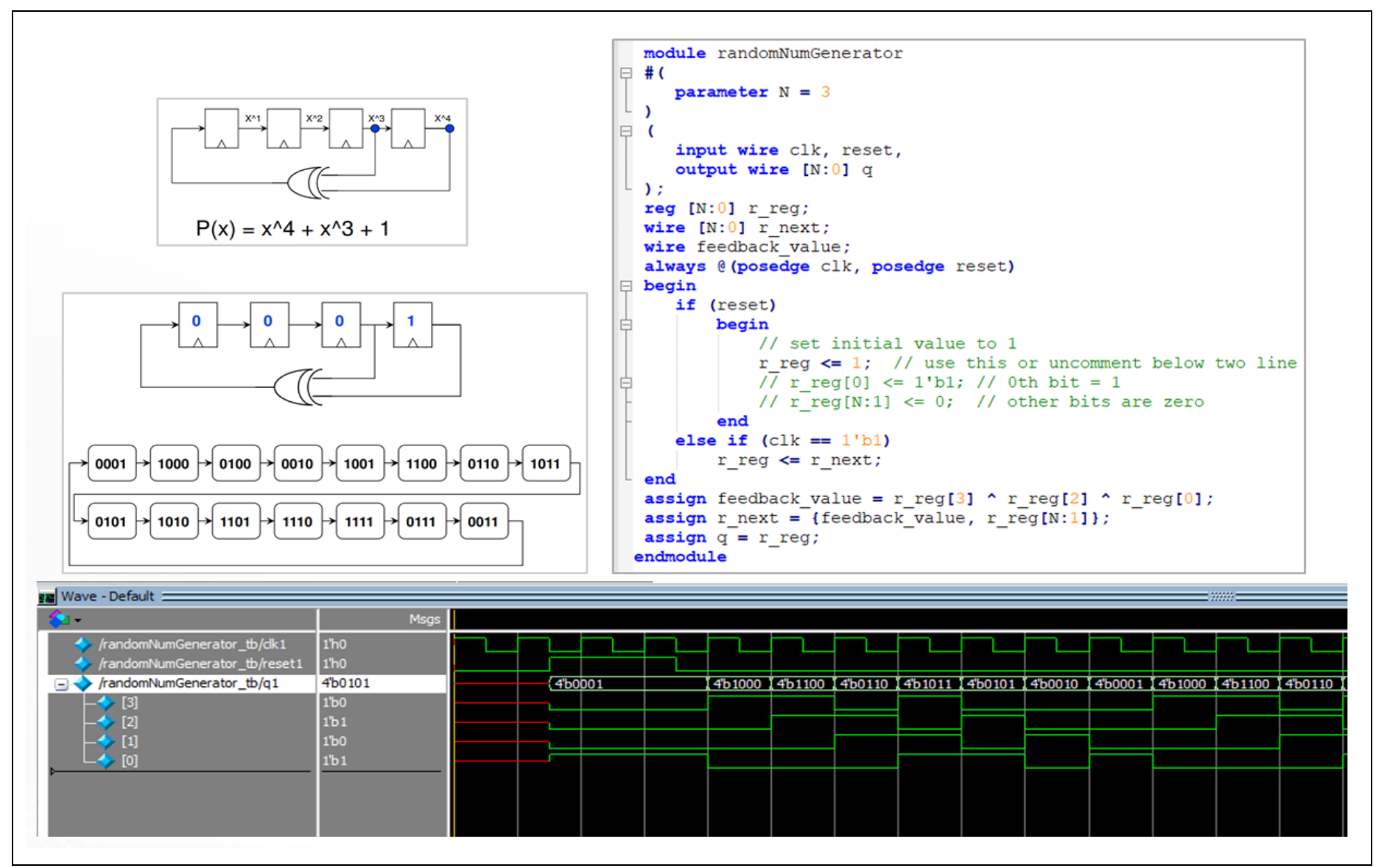

FigURE II: IMPLEMENTATION OF PRNG MODULE BASED ON VERILOG HDL. 


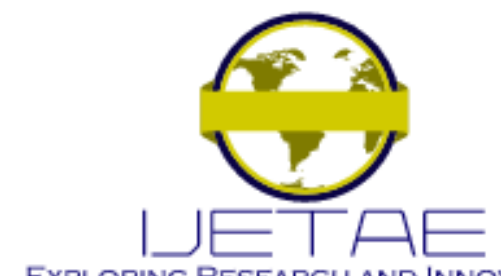

EXPLORING RESEARCH AND INNOVATIONS

International Journal of Emerging Technology and Advanced Engineering

Website: www.ijetae.com (E-ISSN 2250-2459, Scopus Indexed, ISO 9001:2008 Certified Journal, Volume 11, Issue 11, November 2021)

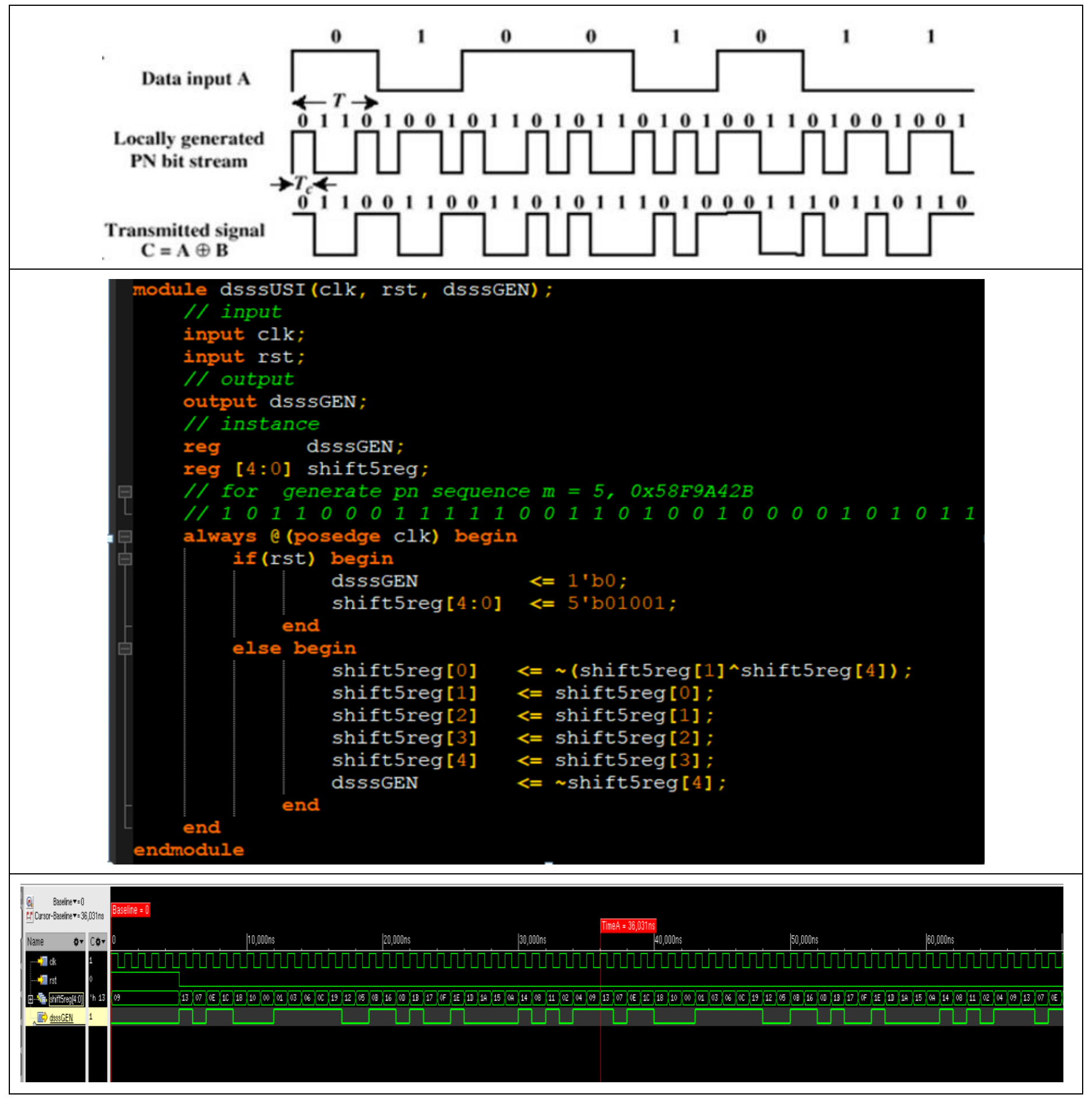

FIGURE III: IMPLEMENTATION OF DSSS SEQUENCE MODULE BASED ON VERILOG HDL. 


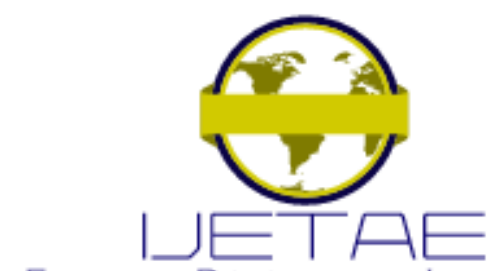

EXPLORING RESEARCH AND INNOVATIONS

International Journal of Emerging Technology and Advanced Engineering

Website: www.ijetae.com (E-ISSN 2250-2459, Scopus Indexed, ISO 9001:2008 Certified Journal, Volume 11, Issue 11, November 2021)

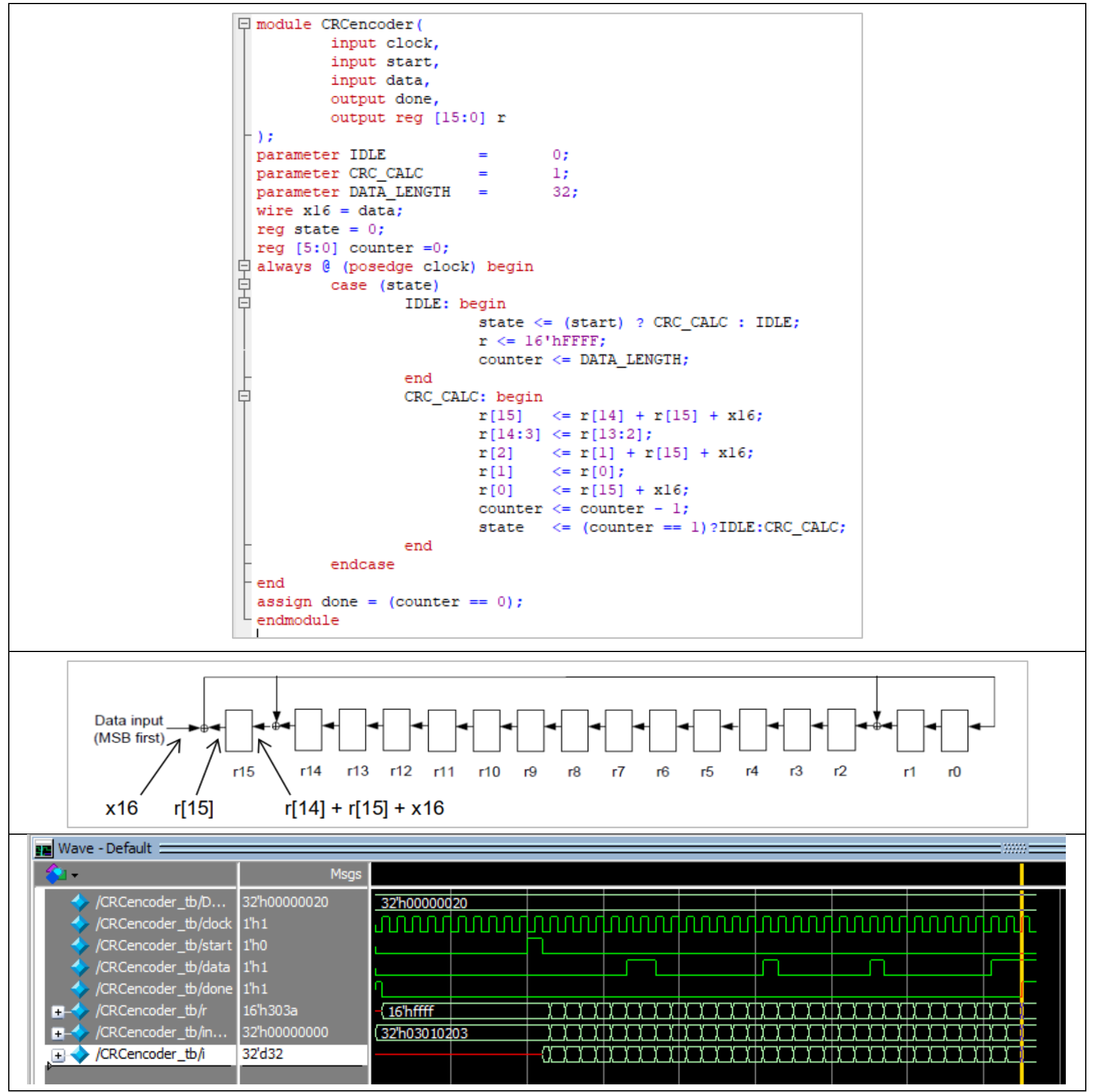

FIGURE IV: Implementation of CRC module based on Verilog HDL. 


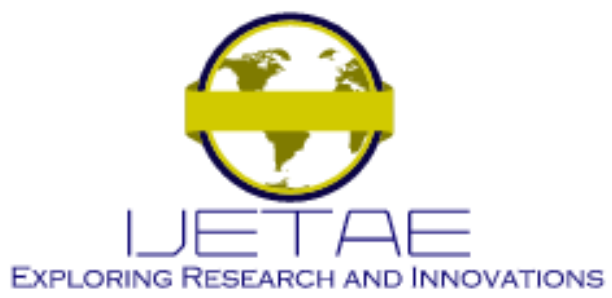

International Journal of Emerging Technology and Advanced Engineering

Website: www.ijetae.com (E-ISSN 2250-2459, Scopus Indexed, ISO 9001:2008 Certified Journal, Volume 11, Issue 11, November 2021)

\section{CONCLUSION}

In this paper, the fundamental concept of LFSR is given. In addition, various applications of LFSR are discussed. It is proved that LFSR can be used in many digital system modules such as processor, BUS, Memory, and DSP. Hence, it is true to say that LFSR is the queen of digital logic functions.

\section{Acknowledgement}

This work is supported by FPT university, Hanoi, Vietnam; and Hutech Institute of Engineering, Ho Chi Minh City University of Technology (HUTECH), Vietnam.

\section{REFERENCES}

[1] Nguyen, D. M. et. al. "New constructions of quantum stabilizer codes based on difference sets". Symmetry 10 (11), 655, 2018.

[2] Nguyen, D. M. et. al. "A novel construction for quantum stabilizer codes based on binary formalism". International Journal of Modern Physics B 34 (8), 2050059, 2020.

[3] Giao, N. P. et. al. "Palm rejection algorithms on touch screen communication system". World Journal of Advanced Engineering Technology and Sciences 2021, 02(02), 052-057

[4] Giao, N. P. et. al. "Fast IQ Amplitude Approximation Method for ASIC Digital System”. International Journal of Engineering Technology and Advanced Engineering, 2021, 11(08), 19-22

[5] Giao, N. P. et. al. "Implementation of Differential Sensing Scheme to Remove Panel Noise in Touchscreen Controller". International Journal of Emerging Technology and Advanced Engineering, 2021, 11 (10), 104-108.

[6] Giao, N. P. et. al. "A Novel Quantum Communication Protocol and its Simulation in IBM Quantum Simulator". International Journal of Emerging Technology and Advanced Engineering, 2021, 11 (7), 8-12. 